\title{
Research Paper \\ Falls and Postural Control in Older Adults with Eye Refractive Errors
}

\begin{abstract}
Afsun Nodehi-Moghadam ${ }^{1,2}$, ${ }^{\text {Maryam Goudarzian }}{ }^{3}$, Farhad Azadi ${ }^{1}$, Seyede Masoumeh Hosseini ${ }^{4}$, Soosan Geranmayeh ${ }^{4}$, Yasin Larni ${ }^{1}$, Maryam Habibi ${ }^{1}$, Pourya Yaghmaei ${ }^{1}$
\end{abstract}

1. Department of Physiotherapy, University of Social Welfare and Rehabilitation Sciences, Tehran, Iran.

2. Iranian Research Center on Ageing, University of Social Welfare and Rehabilitation Sciences, Tehran, Iran.

3. Department of Geritric Nursing, Iranian Research Center of Healthy Aging, Sabzevar University of Medical Sciences, Sabzevar, Iran.

4. Department of Optomtery, School of Rehabilitation Sciences, Iran University of Medical Sciences, Tehran, Iran.

Citation: Nodehi-Moghadam A, Goudarzian M, Azadi F, Hosseini SM, Geranmayeh S, Larni Y, et al. [Falls and postural control in older adults with refractive errors (Persian)]. Iranian Journal of Ageing. 2016; 11(4):126-131. http://dx.doi.org/10.21859/sija-1101126

dol: $:$ http://dx.doi.org/10.21859/sija-1101126

Received: 01 Nov. 2015 Accepted: 05 Jan. 2016

Key words: Balance, Falls, Refractive error, Vision impairment

\section{ABSTRACT}

Objectives The eye refractive errors, including myopia, hyperopia, and astigmatism, are the most important visual impairments worldwide. Based on the existing evidence suggests, the visual impairment could contribute to falling in the elderly. Therefore, this study aimed to determine the influence of vision impairment of older adults with eye refractive errors on the occurrence of falls and postural control. Methods \& Materials The older people referred to the health center of the 9th district of Tehran municipality for ophthalmic examination were examined (using ophthalmoscope and Snellen chart). Based on eye screening results, 77 older adults with refractive errors of hyperopia $(n=19$, mean [SD] age $=67$ [5.85] y); astigmatism ( $n=22$, mean [SD] age $=70.32[5.60]$ y); and both hyperopia and astigmatism ( $n=36$, mean [SD] age $=69.78$ [7.69] y), as well as 23 older healthy adults, mean (SD) age $=70.29(5.29) \mathrm{y}$, without any obvious eye disorders were enrolled in this study. Their history of falls and postural control were evaluated. The postural control was determined using the Timed Up and Go (TUG) test. The statistical tests were analysis of variance (ANOVA) for comparing the groups with regard to postural control and the Chi-square test for comparing the prevalence of falls between groups with refractive errors and the healthy group.

Results The studied subjects comprised $23 \%(n=23)$ healthy ones, $19 \%(n=19)$ people with hyperopia, $22 \%(n=22)$ people with astigmatism, and $36 \%(n=36)$ people with both hyperopia and astigmatism. About $70 \%$ of them were women, and $30 \%$ were men. The results of our study showed that $15.8 \%$ of study participants with normal vision, $26.1 \%$ with hyperopia, $22.7 \%$ with astigmatism, and $38.9 \%$ with both hyperopia and astigmatism had experienced at least 2 falls in the past 12 months. The people with refractive errors showed a higher prevalence of falls; however, the result of the Chi-square test did not reveal any significant differences among these groups $(P=0.25)$. Furthermore, the results of ANOVA for comparing TUG test (test of postural control) results showed no differences among these groups $(\mathrm{P}=0.64)$.

Conclusion Vision impairment of older adults due to refractive error is not associated with an increase in falls. Furthermore, TUG test results did not show balance disorders in these groups. Further research, such as assessment of postural control with advanced devices and considering other falling risk factors is also needed to identify the predictors of falls in older adults with eye refractive errors.

\section{* Corresponding Author:}

Maryam Goudarzian, MSc.

Address: Department of Geriatric Nursing, Iranian Research Center of Healthy Aging, Sabzevar University of Medical Sciences, Sabzevar, Iran. Tel: +98 (935) 5389867

E-mail: mary_ac_ir@yahoo.com 


\title{
زمينخوردن و كتترل ياسجرال در سالمندان با عيوب انكسارى خشم
}

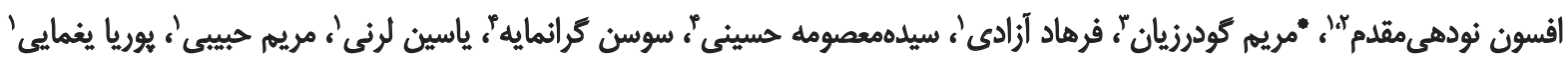

\author{
1- كروه فيزيوترائى، دانشكاه علوم بهزيستى و توانبخشى، تهران، ايران.

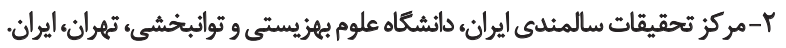

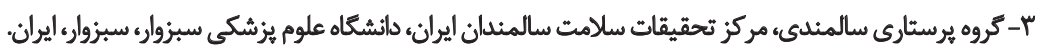

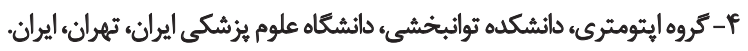

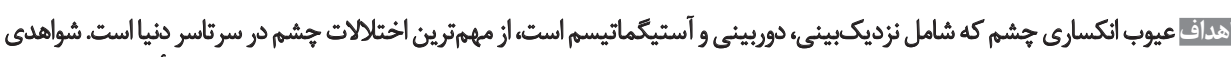

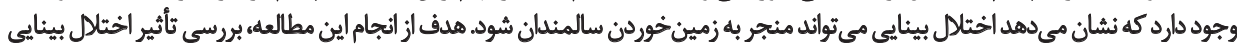

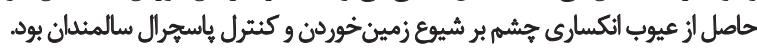

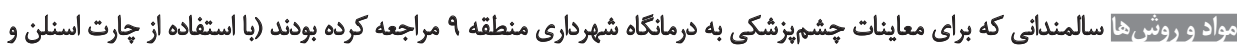

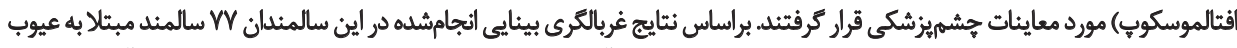

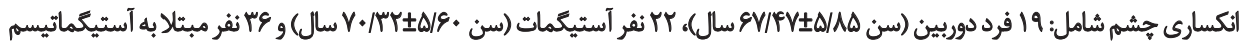
همراه دوربينى (سن

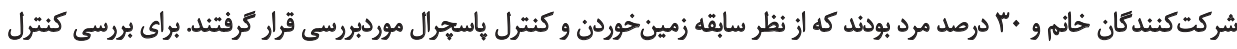

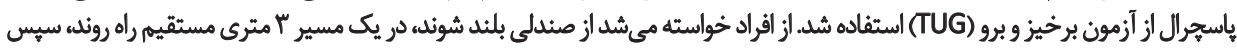

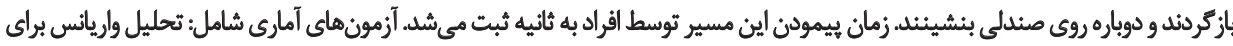

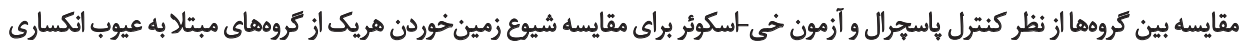
نسبت به كروه كنترل سالم بوده.

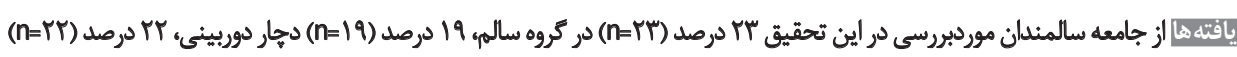

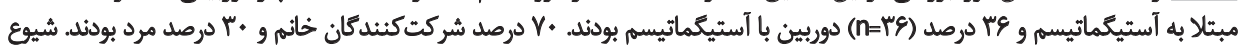

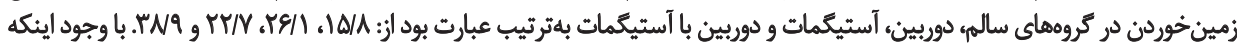

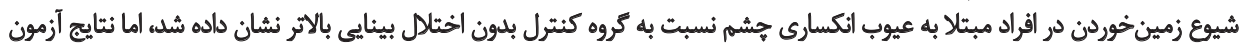

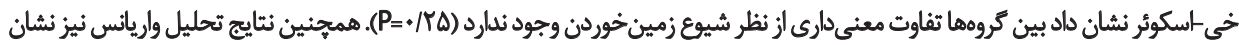

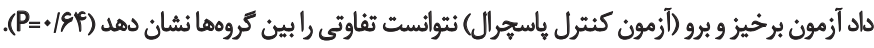

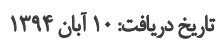

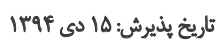

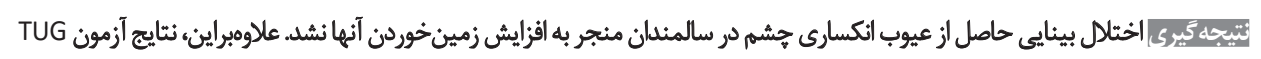

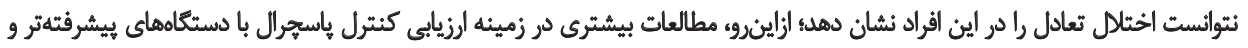

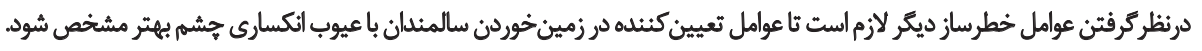

كليدوازوها:

تعادل، زمين خورن

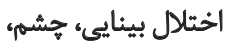
عيوب أنكسارى بينايع، جئ

آستيكماتيسم "است، از مههمترين اختلالات جشمه در سراسر دنيا

مقدمه

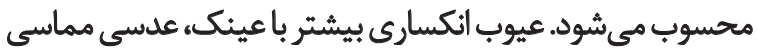

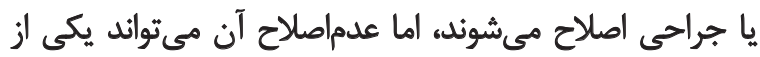
عيوب انكسارى جشم' كه شامل نزديكبينى '، دوربينى" و

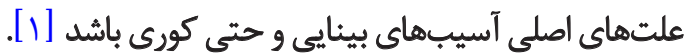
مرور متون كذشته نشان ميدهد در بيشتر نقاط دنيا در

1. Refractive error

2. Myopia

3. hyperopia 


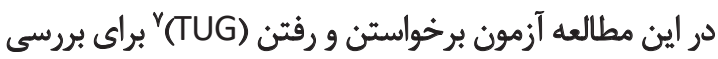

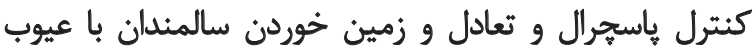

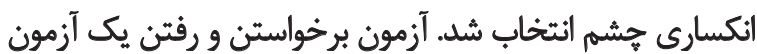

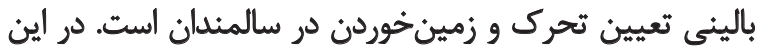

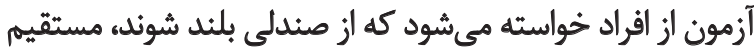

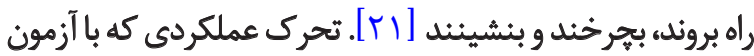

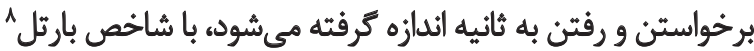

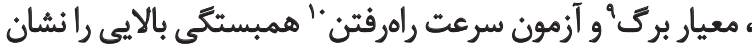

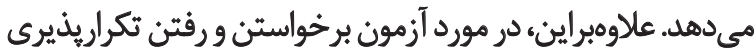

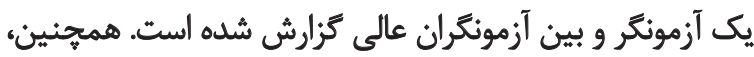

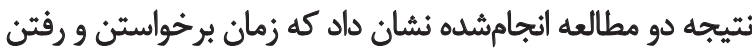

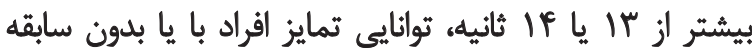

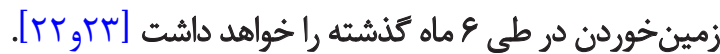

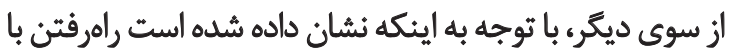

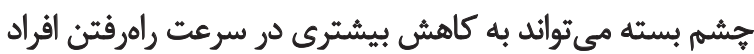

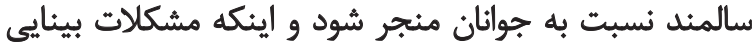

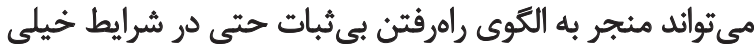

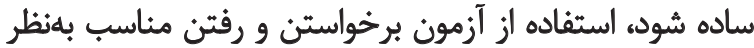

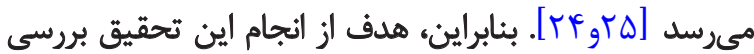

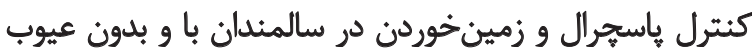

$$
\text { انكسارى جشم است. }
$$

\section{روش مطالعه}

سالمندان موردبررسى از بين افرادى كه توسط بيزشك

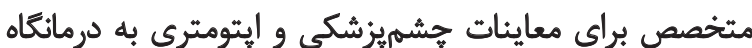

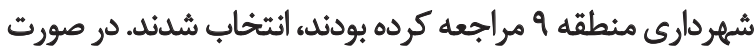

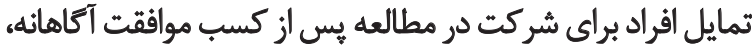

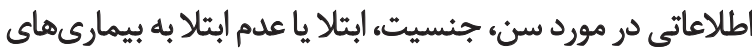

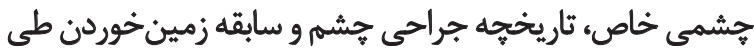

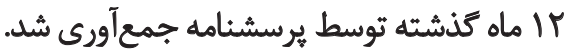

معيارهاي ورود به مطالعه عبارت بودند ازئ سن ه \& سال يا

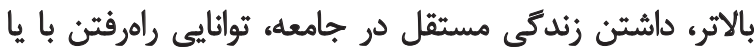

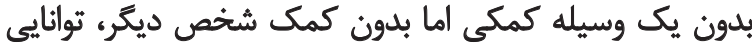

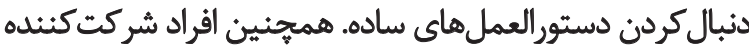

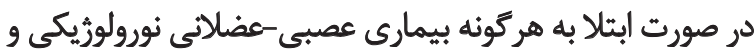

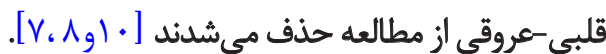

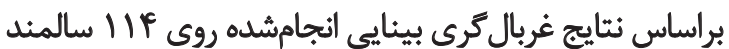
مراجعلكنيده به درمانكاه منطقه 9 شهرداري، شايعترين

\section{Timed up and go}

8. Barthel index of activities of daily living

9. Berg

10. Gait speed testing

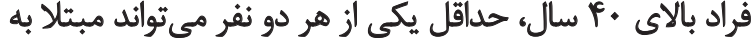

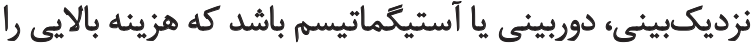

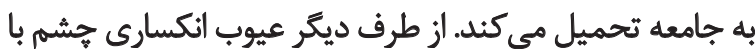

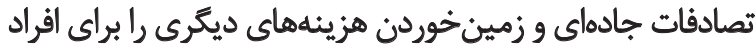

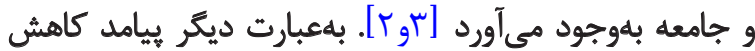

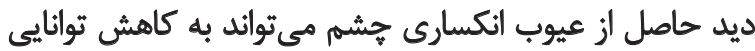

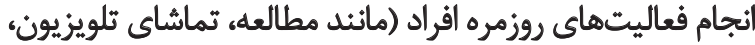

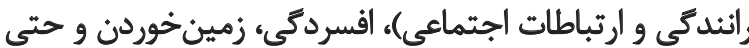
افزايش مركومير منجر شود [ه-ب].

زمينخوردن مسئلداى مهم در سلامت عمومى و مهمثترين

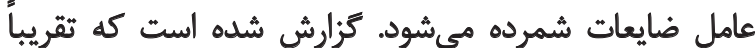

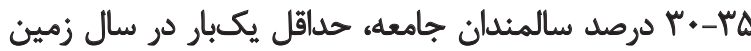

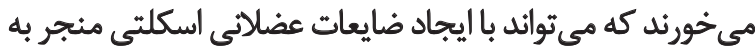

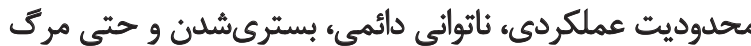
افراد سالمند شود [9-9].

توانايى كنترل بدن در فضا (كنترل ياسجرال) ه و مركز ثقل

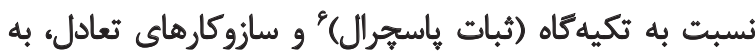

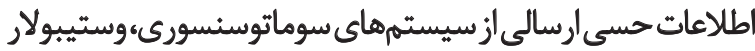

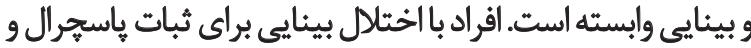

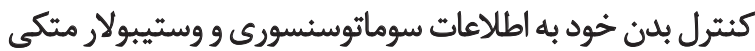

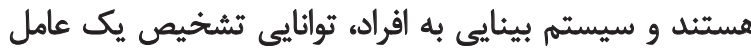

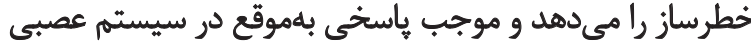

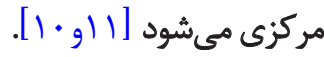
مطالعات زيادى در مورد بررسى ارتباط بين اختلال بينايى

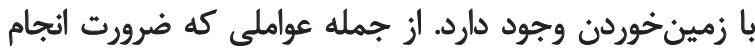

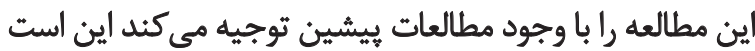
كه در متون كذشته درباره ارتباط بين اختلال بينايى و أفرايش

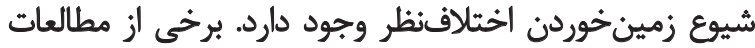

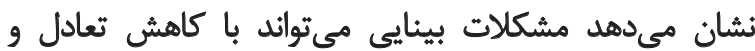

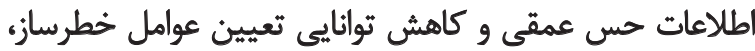

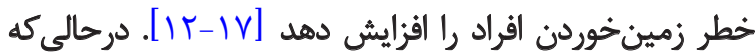

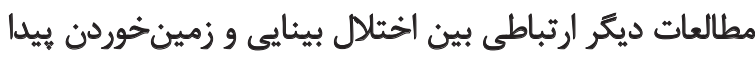

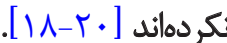

علاوهبراين، انجام تحقيق حاضر به اين دليل ضرورى است كه

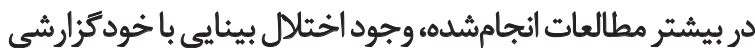

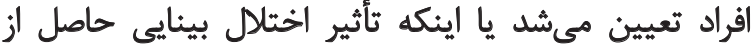

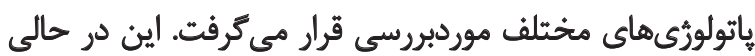

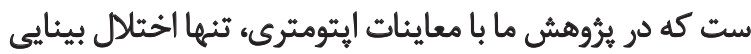
حاصل از عيوب انكسارى جشم برم بررسى شد.

5. Postural control

6. Postural stability 
نتايج تحليل واريائس نيز نشان داد كه آزمون برخواستن و رفتن نفائن

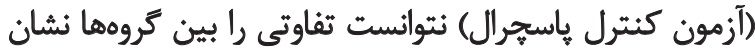

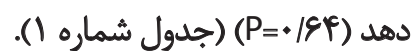

ث

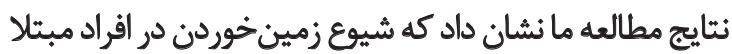

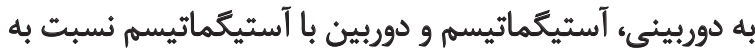

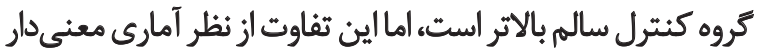

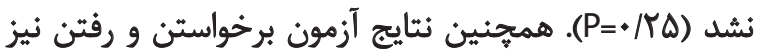

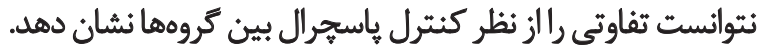
شاموى كوك" و همكارانش [rr] نشان دادند كه برخواستن

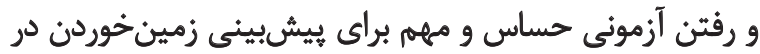

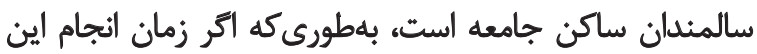

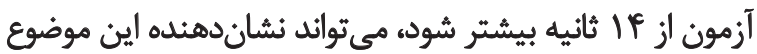

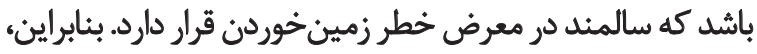

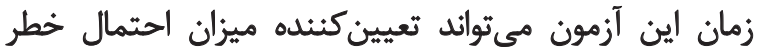

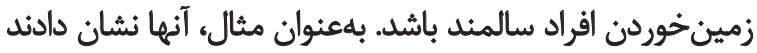

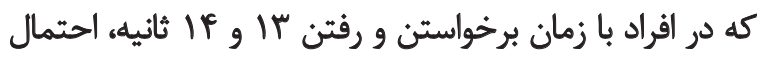
زمين

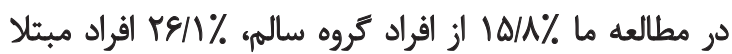

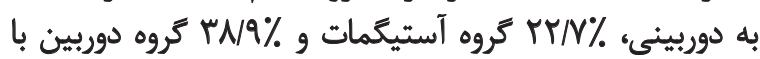

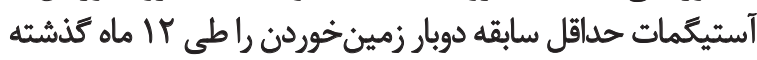

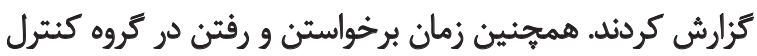

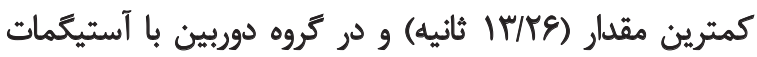

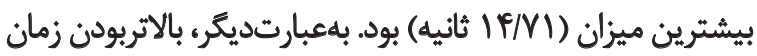

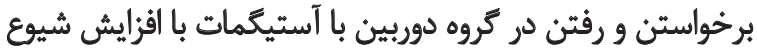

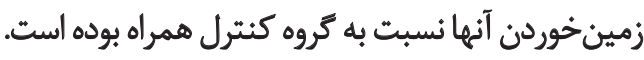
در متون كذشته در مورد ارتباط بين دقت بينايى" با كنترل

12. Shumway-Cook

13. Visual acuity
عيوب انكسارى تشخيصدادهده در سالمندان، دوربينى و

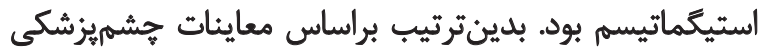

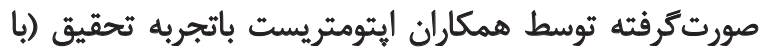

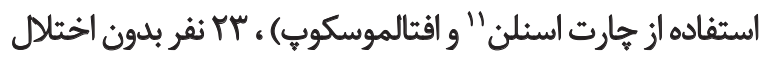

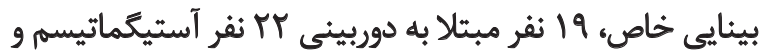

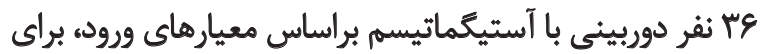

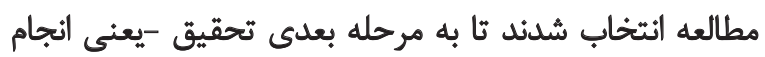
آزمون كنترل ياسجرال (آزمون برخواستن و رفتن بعدي) - وارد شوند.

$$
\text { نحوه انجام أزمون بوخواستن و رقتن }
$$

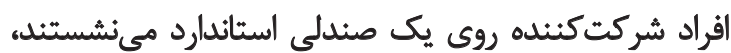

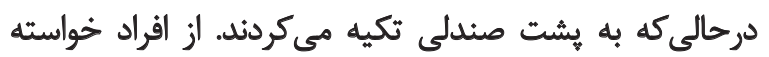

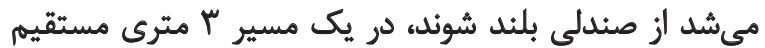

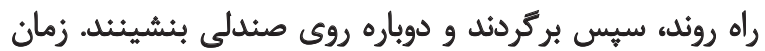

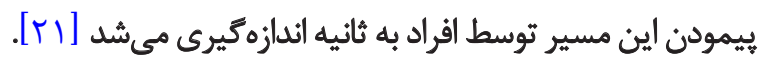

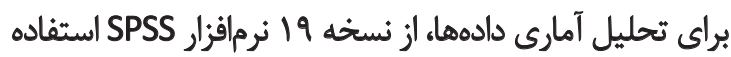

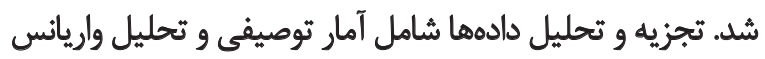

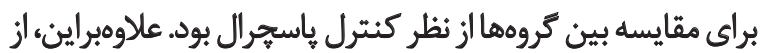

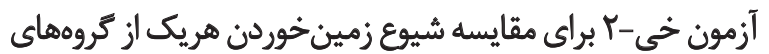
مبتلا به عيوب انكسارى نسبت به كروه كنترل سالم استفاده شد.

ياثتهها

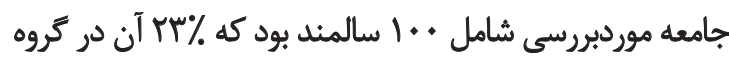

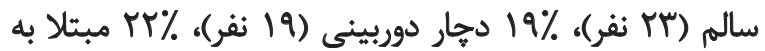

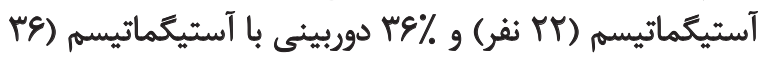

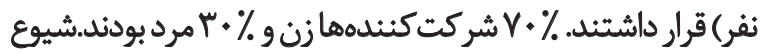

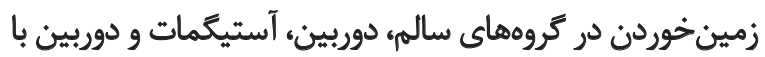

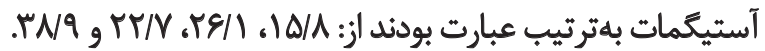

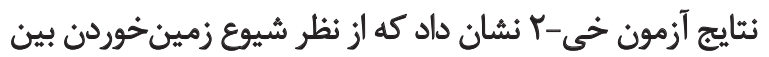

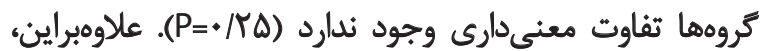

11. Snellen's illiterate E chart

جدول ا. مقايسه كنترل ياسجرال و شيوع زمين خوردن در افراد مبتلا به عيوب ائكسارى جشمه.

\begin{tabular}{|c|c|c|c|}
\hline \multicolumn{3}{|c|}{ ميانكين ثلاتحر|ف معيار } & \multirow{2}{*}{$\log 5$} \\
\hline زمان آزهون برخواستن و رقتن & ميزان زمين خوردن & سن سن & \\
\hline $\mid r / K g(r / W g)$ & $10 / 1 \%$ & $V \cdot / R q \pm \Delta / F q$ & طبيعي \\
\hline $\mid f / \Delta v(\varphi / .9)$ & rell\% & $Q V / P V \pm \Delta / \Delta \Lambda$ & دوربين \\
\hline$|f /| q(\varphi / .9)$ & $M T / V \%$ & $\gamma \cdot / r Y \pm \Delta / \varepsilon$. & أسثيكمات \\
\hline $\mid f / N(\Delta / / A)$ & rNA\% & $e q / V A \pm V / \varepsilon q$ & دوريين+أستيكمات \\
\hline.$/ a t$ & . RQ &.$/ \% \Delta$ & P-value \\
\hline
\end{tabular}

L 


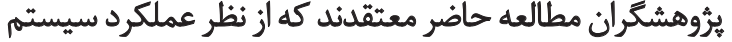

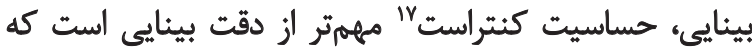

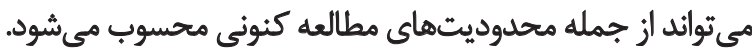

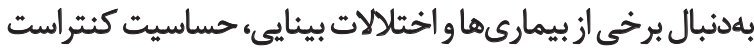

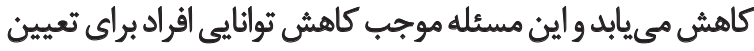

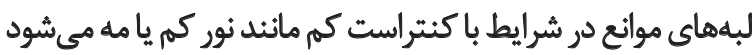

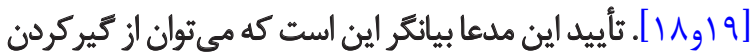

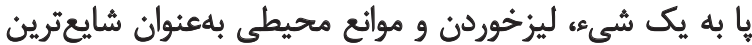

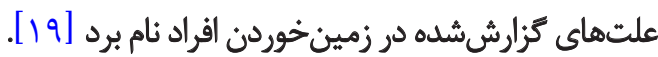
مطالعه كنونى، محدوديتهاى ديخرى نيز دارد. آنغونه

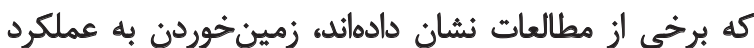

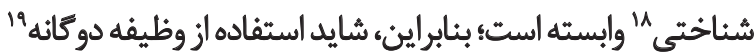

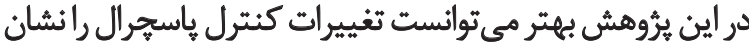

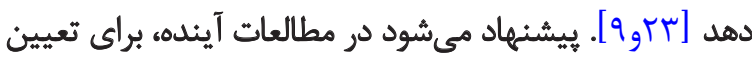

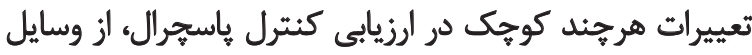
آزمايشعاهي دقيق و حساستر استفاده شود.
باسجرال و افزايش شُيع زمينخوردن سالمندان توافقى وجود

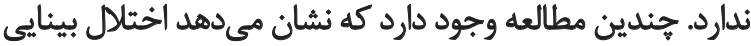

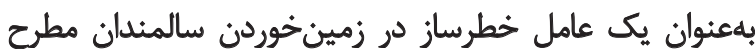

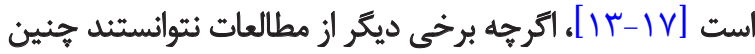

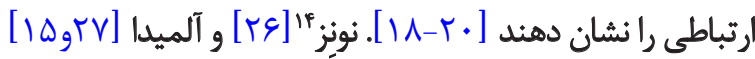

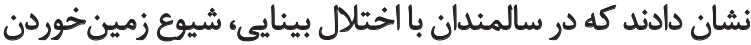

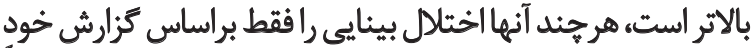

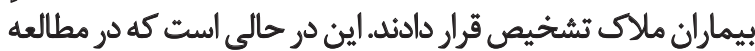

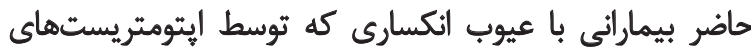
باتجربه موردبررسي قرار كرفته بودند، شركت بـ داشتيند.

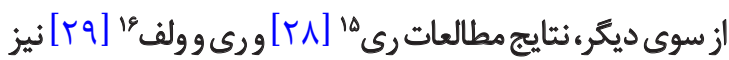

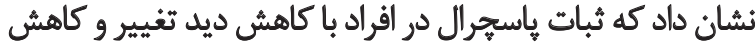

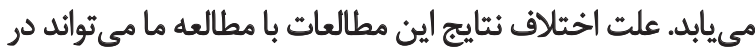

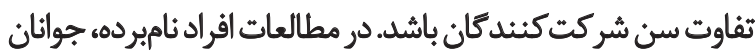

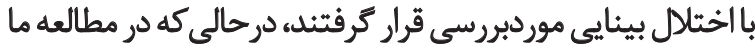

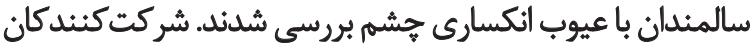

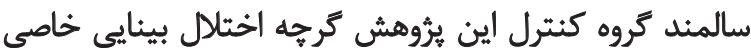

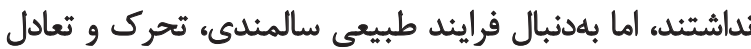

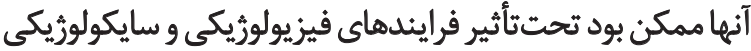
طبيعى، دستخوش تغييراتى شده باشيد.

مطالعات زيادى وجود دارد كه نشان ميدهد بهاطورطبيعى با ماند سالمندى، قدرت عضلاتى و دامنه حركتى مفاصل كاهش مئ مي يادي

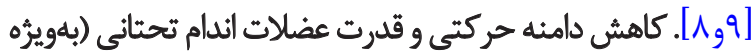

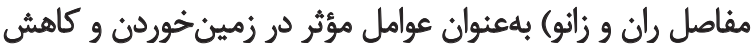

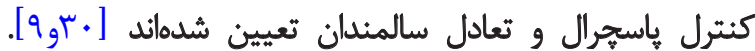

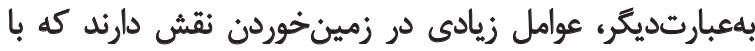

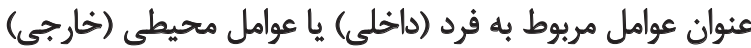

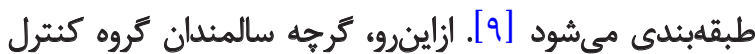

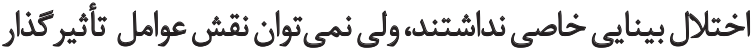

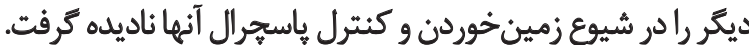

\section{نتيجهلكيرى نهايي}

اختلال بينايى حاصل از عيوب انكسارى جشم در سالمندان،

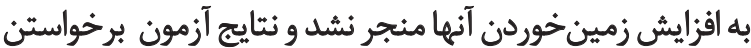

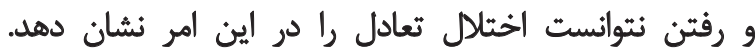

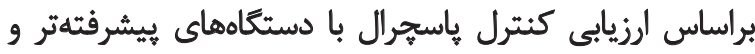

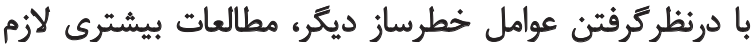

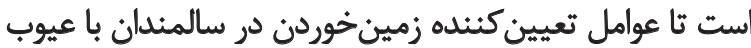

$$
\text { انكسارى جشهم، بهتر مشخص شول شود. }
$$

14. Nunes

15. Ray

16. Ray and Wolf 


\section{References}

[1] Verhoeven VJ, Wong KT, Buitendijk GH, Hofman A, Vingerling JR, Klaver CC. Visual consequences of refractive errors in the general population. Ophthalmology. 2015; 122(1):101-9.

[2] Yekta AA, Hashemi H, Ostadimoghaddam H, Shafaee Sh, Norouzirad R, Khabazkhoob M. Prevalence of Refractive Errors among the Elderly Population of Sari, Iran. Iranian Journal of Ophthalmology. 2013; 25(2):123-132.

[3] Desapriya E, Subzwari S, Scime-Beltrano G, Samayawardhena LA, Pike I. Vision improvement and reduction in falls after expedited cataract surgery: Systematic review and metaanalysis. Journal of Cataract \& Refractive Surgery. 2010; 36(1):13-9.

[4] Polack S, Kuper H, Wadud Z, Fletcher A, Foster A. Quality of life and visual impairment from cataract in Satkhira district, Bangladesh. British Journal of Ophthalmology. 2008; 92(8):1026-30.

[5] Polack S, Eusebio C, Fletcher A, Foster A, Kuper H. Visual impairment from cataract and health related quality of life: Results from a case-control study in the Philippines. Ophthalmic Epidemiology. 2010; 17(3):152-9.

[6] Lopez D, McCaul KA, Hankey GJ, Norman PE, Almeida OP, Dobson AJ, et al. Falls, injuries from falls, health related quality of life and mortality in older adults with vision and hearing impairment-Is there a gender difference? Maturitas. 2011; 69(4):359-64

[7] Kuang TM, Tsai SY, Hsu WM, Cheng CY, Liu JH, Chou P. Visual impairment and falls in the elderly: The Shihpai Eye Study. Journal of the Chinese Medical Association. 2008; 71(9):467-72.

[8] Hu J, Xia Q, Jiang Y, Zhou P, Li Y. Risk factors of indoor fall injuries in community-dwelling older women: A prospective cohort study. Archives of Gerontology and Geriatrics. 2015; 60(2):259-64.

[9] Ambrose AF, Paul G, Hausdorff JM. Risk factors for falls among older adults: A review of the literature. Maturitas. 2013; 75(1):51-61.

[10] Horak FB. Postural orientation and equilibrium: what do we need to know about neural control of balance to prevent falls? Age and Ageing. 2006; 35(2):7-11.

[11] Riemann BL, Lephart SM. The sensorimotor system, part II: The role of proprioception in motor control and functional joint stability. Journal of Athletic Training. 2002; 37(1):80-4.

[12] Bugnariu N, Fung J. Aging and selective sensorimotor strategies in the regulation of upright balance. Journal of Neuroengineering and Rehabilitation. 2007; 4(1):1-19.

[13] Lord SR, Menz HB. Visual contributions to postural stability in older adults. Gerontology. 2000; 46(6):306-10.

[14] Freeman EE, Munoz B, Rubin G, West SK. Visual field loss increases the risk of falls in older adults: The Salisbury eye evaluation. Investigative Ophthalmology \& Visual Science. 2007; 48(10):4445-50.

[15] Boptom RQ, Cumming RG, Mitchell P, Attebo K. Visual impairment and falls in older adults: The Blue Mountains Eye Study. Journal of the American Geriatrics Society. 1998; 46(1):58-64.

[16] Coleman AL, Stone K, Ewing SK, Nevitt M, Cummings S, Cauley JA, et al. Higher risk of multiple falls among elderly women who lose visual acuity. Ophthalmology. 2004; 111(5):857-62.
[17] Reed-Jones RJ, Solis GR, Lawson KA, Loya AM, Cude-Islas D, Berger CS. Vision and falls: A multidisciplinary review of the contributions of visual impairment to falls among older adults. Maturitas. 2013; 75(1):22-8.

[18] de Boer MR, Pluijm SM, Lips P, Moll AC, Völker-Dieben HJ, Deeg DJ, et al. Different aspects of visual impairment as risk factors for falls and fractures in older men and women. Journal of Bone and Mineral Research. 2004; 19(9):1539-47.

[19] Lord SR, Clark RD, Webster IW. Visual acuity and contrast sensitivity in relation to falls in an elderly population. Age and Ageing. 1991; 20(3):175-81.

[20] Lamoureux E, Gadgil S, Pesudovs K, Keeffe J, Fenwick E, Dirani $\mathrm{M}$, et al. The relationship between visual function, duration and main causes of vision loss and falls in older people with low vision. Graefe's Archive for Clinical and Experimental Ophthalmology. 2010; 248(4):527-33

[21] Podsiadlo D, Richardson S. The timed "Up \& Go": A test of basic functional mobility for frail elderly persons. Journal of the American Geriatrics Society. 1991; 39(2):142-8.

[22] Kristensen MT, Foss NB, Kehlet H. Timed "up \& go" test as a predictor of falls within 6 months after hip fracture surgery. Physical Therapy. 2007; 87(1):24-30.

[23] Shumway-Cook A, Brauer S, Woollacott M. Predicting the probability for falls in community-dwelling older adults using the Timed Up \& Go Test. Physical Therapy. 2000; 80(9):896-903.

[24] Elliott DB, Bullimore MA, Patla AE, Whitaker D. Effect of a cataract simulation on clinical and real world vision. British Journal of Ophthalmology. 1996; 80(9):799-804.

[25] Helbostad JL, Vereijken B, Hesseberg K, Sletvold O. Altered vision destabilizes gait in older persons. Gait \& Posture. 2009; 30(2):233-8.

[26] Nunes BP, de Oliveira Saes M, Siqueira FV, Tomasi E, Silva SM, da Silveira DS, et al. Falls and self-assessment of eyesight among elderly people: A population-based study in a south Brazilian municipality. Archives of Gerontology and Geriatrics. 2014; 59(1):131-5.

[27] Almeida ST, Soldera CL, Carli GA, Gomes I, Resende TD Analysis of extrinsic and intrinsic factors that predispose elderly individuals to fall. Revista da Associação Médica Brasileira. 2012 58(4):427-33.

[28] Ray CT, Horvat M, Croce R, Mason RC, Wolf SL. The impact of vision loss on postural stability and balance strategies in individuals with profound vision loss. Gait \& Posture. 2008; 28(1):58-61.

[29] Ray CT, Wolf SL. Gender differences and the risk of falls in individuals with profound vision loss. Journal of Visual Impairment \& Blindness. 2010; 104(5):311-16.

[30] Nodehi-Moghadam A, Taghipour M, Alibazi RG, Baharlouei $\mathrm{H}$. The comparison of spinal curves and hip and ankle range of motions between old and young persons. Medical Journal of the Islamic Republic of Iran. 2014; 28(1):74-80. 